\title{
Erratum
}

\section{Erratum to "Magnetic Resonance Imaging of Atherosclerosis Using CD81-Targeted Microparticles of Iron Oxide in Mice"}

\author{
Fei Yan (id, ${ }^{1,2,3}$ Wei Yang, ${ }^{2}$ Xiang Li, ${ }^{2}$ Hongmei Liu, ${ }^{1}$ Xiang Nan, ${ }^{2}$ Lisi Xie, ${ }^{2}$ Dongliang Zhou, \\ Guoxi Xie, ${ }^{2}$ Junru Wu $\mathbb{i},{ }^{4}$ Bensheng Qiu, ${ }^{2}$ Xin Liu, ${ }^{2}$ and Hairong Zheng $\mathbb{1}^{1,2}$ \\ ${ }^{1}$ The Third Affiliated Hospital of Southern Medical University, Guangzhou 510500, China \\ ${ }^{2}$ Paul C. Lauterbur Research Center for Biomedical Imaging, Institute of Biomedical and Health Engineering, \\ Shenzhen Institutes of Advanced Technology, Chinese Academy of Sciences, Shenzhen 518055, China \\ ${ }^{3}$ Shenzhen Key Laboratory for Molecular Imaging, Shenzhen 518055, China \\ ${ }^{4}$ Department of Physics, University of Vermont, Burlington, VT 05405, USA \\ Correspondence should be addressed to Hairong Zheng; hr.zheng@siat.ac.cn
}

Received 29 August 2018; Accepted 5 September 2018; Published 18 October 2018

Copyright (C) 2018 Fei Yan et al. This is an open access article distributed under the Creative Commons Attribution License, which permits unrestricted use, distribution, and reproduction in any medium, provided the original work is properly cited.

In the article titled "Magnetic Resonance Imaging of Atherosclerosis Using CD81-Targeted Microparticles of Iron Oxide in Mice" [1], the images in the IgG-MPIO column in Figure 5 did not clearly show the difference between the movement before and after injection. Therefore, they have been replaced with a locally enlarged higher-resolution version in place.

In addition, there was an error in the legend of Figure 5 where " $(P<0.05$ at 14 weeks; $P<0.01$ at 20 and 30 weeks)", "(mean $\pm S D)$, with no significant difference in postMPIO CNR between time-points. Scale bars $=1 \mathrm{~mm}$ ", and "at baseline and 30 and 60 minutes after injection of PV-MPIO" should be removed. The corrected Figure and legend are as follows. 

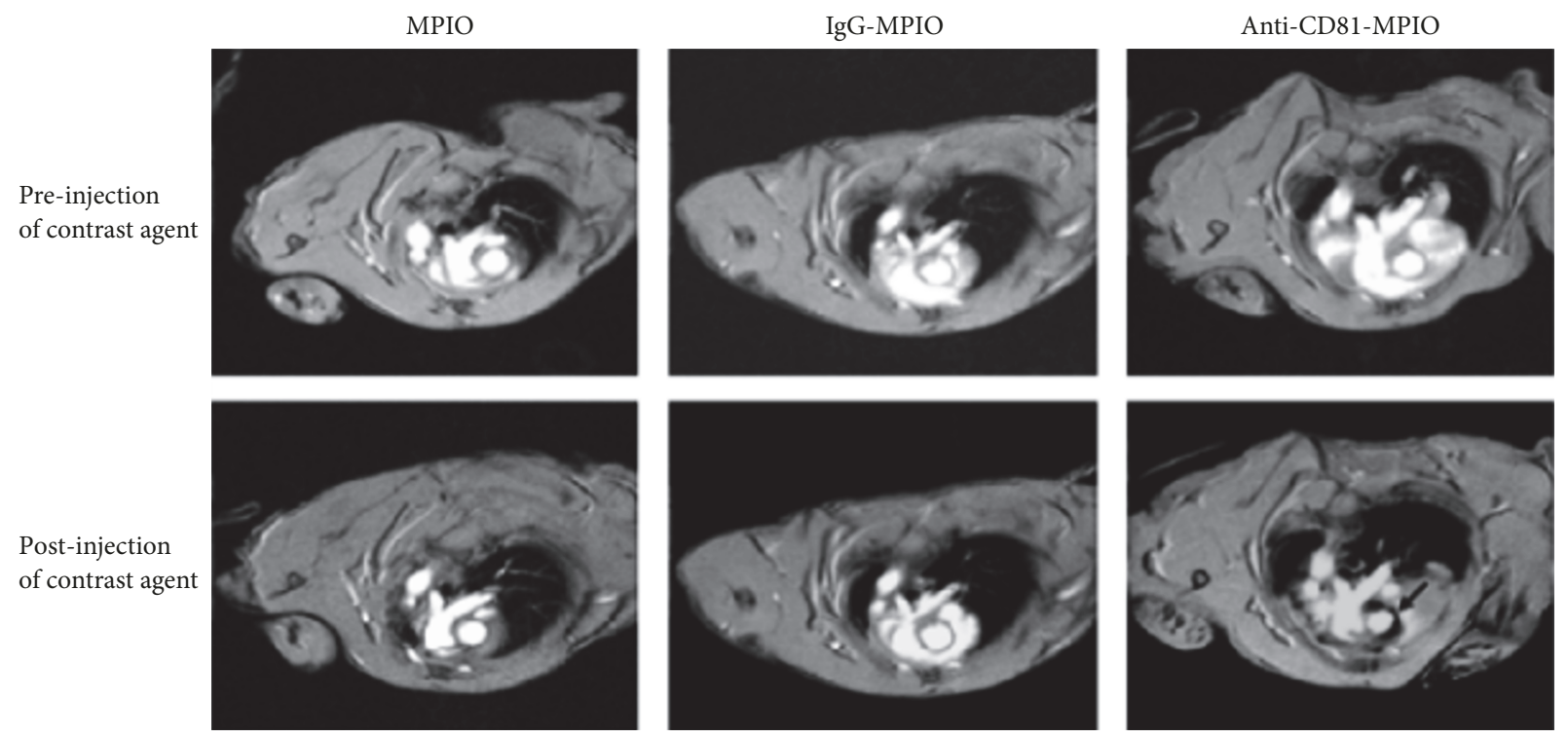

(a)
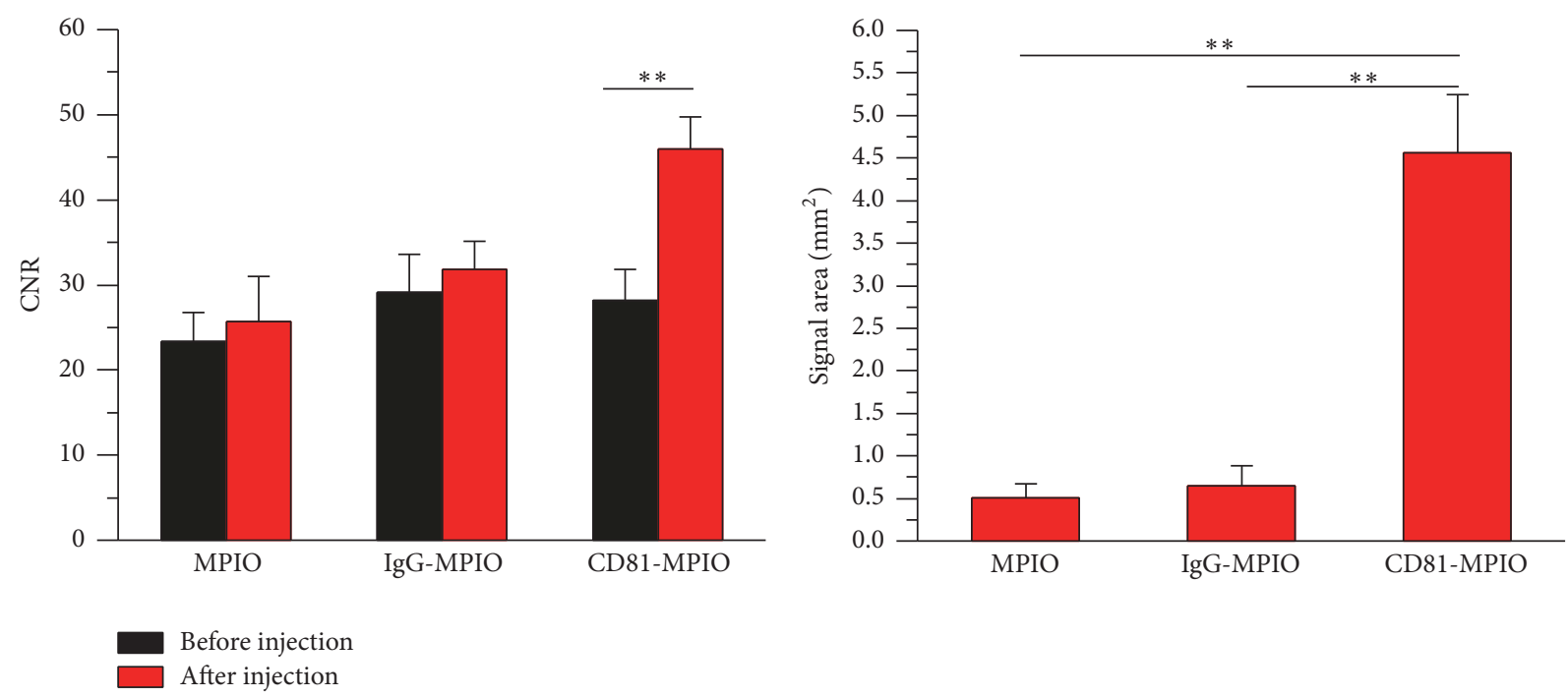

(b)

(c)

FIGURE 5: In vivo MR images of aortic root after MPIO injection.(a) Representative MR images of the aortic root in apoE-/- mice before or after injection with MPIO (left), IgG-MPIO (middle), or CD81-MPIO (right). The arrow points out the low signal areas after CD81MPIO injection. (b) Contrast-to-noise ratio (CNR) of MPIO-positive lesion areas was significantly increased after injection of CD81MPIO compared to equivalent lesion areas on precontrast images. (c) Quantitative analysis of MRI data. Mean area ( \pm SD) of low MR signal areas in aortic roots.

\section{References}

[1] F. Yan, W. Yang, X. Li et al., "Magnetic resonance imaging of atherosclerosis using CD81-targeted microparticles of iron oxide in mice," BioMed Research International, vol. 2015, Article ID 758616, 10 pages, 2015. 


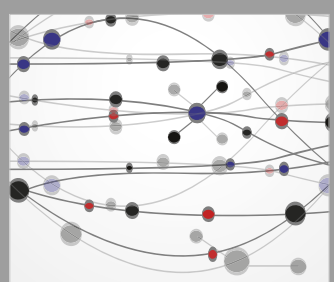

The Scientific World Journal
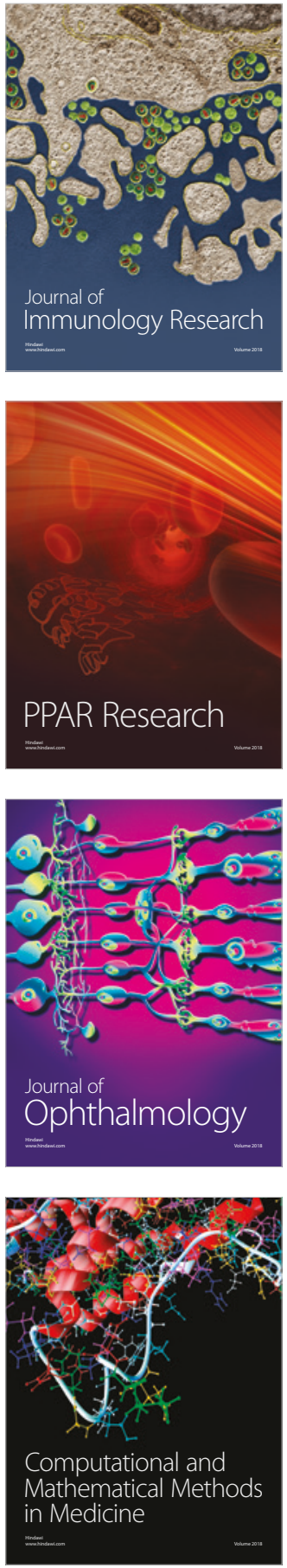

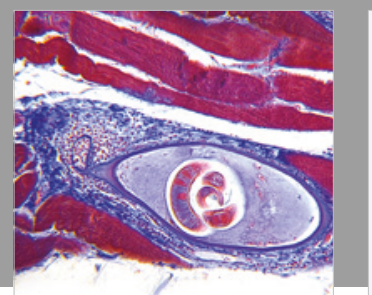

Gastroenterology Research and Practice

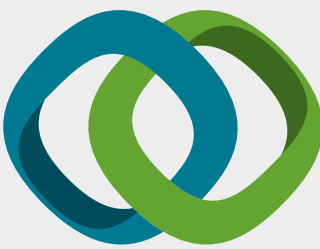

\section{Hindawi}

Submit your manuscripts at

www.hindawi.com
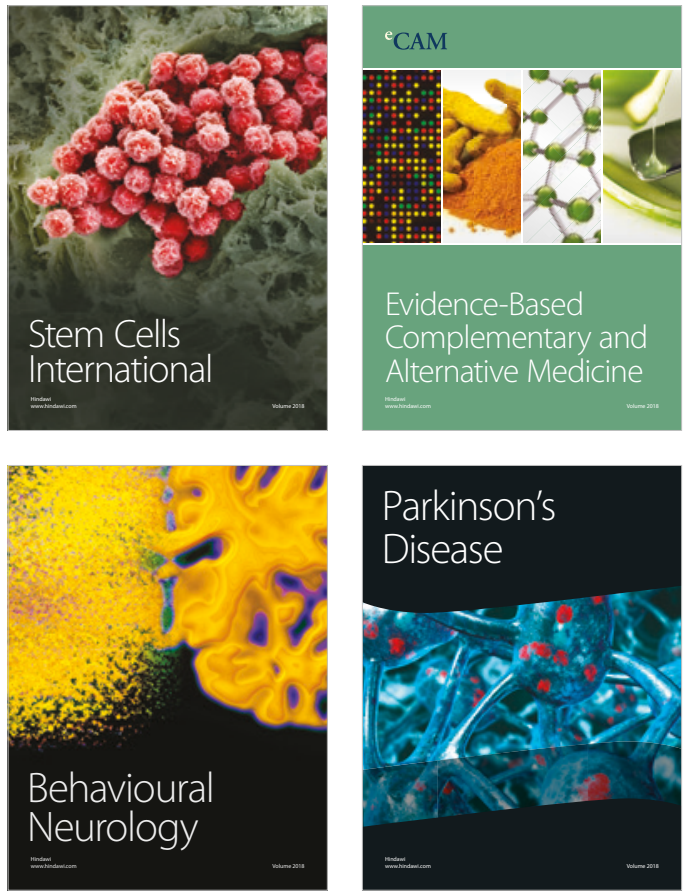

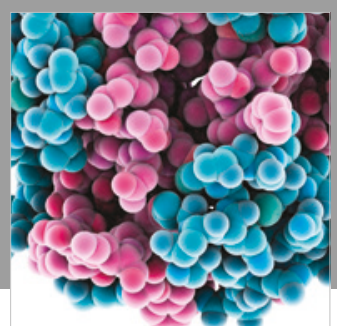

ournal of

Diabetes Research

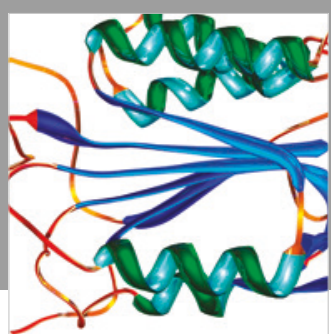

Disease Markers
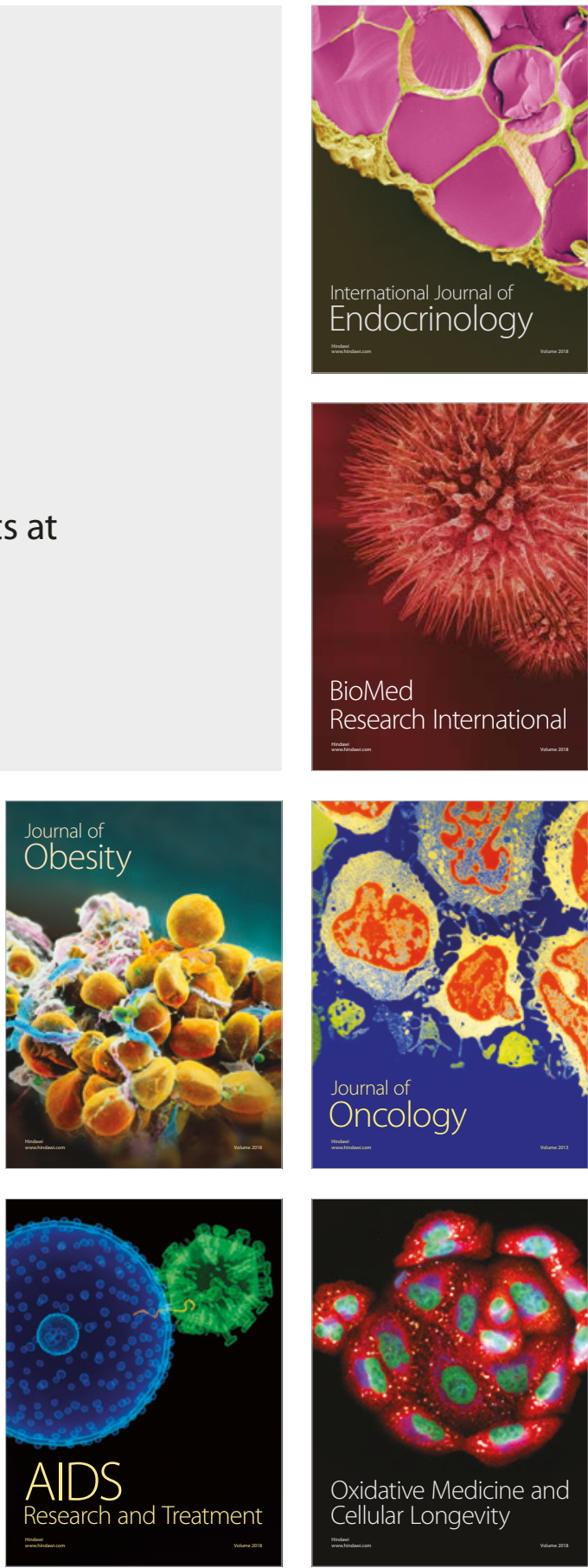